\title{
A concept of sincerity for combinatorial voting
}

\author{
Francesco De Sinopoli · Claudia Meroni
}

Received: date / Accepted: date

\begin{abstract}
A basic problem in voting theory is that all the strategy profiles in which nobody is pivotal are Nash equilibria. We study elections where voters decide simultaneously on several binary issues. We extend the concept of conditional sincerity introduced by Alesina and Rosenthal (1996) and propose an intuitive and simple criterion to refine equilibria in which players are not pivotal. This is shown to have a foundation in a refinement of perfection that takes into account the material voting procedure. We prove that in large elections the proposed solution is characterized through a weaker definition of Condorcet winner and always survives sophisticated voting.
\end{abstract}

Keywords Voting theory $\cdot$ Multi-issue elections - Strategic voting $\cdot$ Perfect equilibrium

JEL Classification C72 - D72

\section{Introduction}

Democracy can be realized through two basic forms, the representative one and the direct one. In a representative democracy people elect government officials and/or parliamentary members who exercise the political power taking final decisions. On the contrary, in a direct democracy people participate in the decision making personally. Modern democracies are typically representative, nevertheless some forms of direct democracy still persist, mostly through referenda. When such a form of democracy is carried out several issues are often proposed together, so that citizens

We thank the associate editor and two anonymous referees for their comments.

F. De Sinopoli · C. Meroni

Department of Economics, University of Verona, via Cantarane 24, 37129 Verona, Italy

E-mail: francesco.desinopoli@univr.it

C. Meroni $(\bowtie)$

E-mail: claudia.meroni@univr.it 
express their preferences on those issues in the same ballot. ${ }^{1}$ If the referendum is just on a single issue the theory unambiguously tells us that each citizen should express her preferences sincerely, because this is her dominant strategy. Things become much more obscure in case multiple referenda are held simultaneously. In this paper, we try to shed some light on the problems that arise in such a situation and we propose a solution in the same spirit as in the single-issue setting.

In case an elector is called to vote on multiple issues she may have non-separable preferences on them, meaning that she may prefer that a given issue passes or not depending on the resolution of the others. ${ }^{2}$ This is the source of the difficulties in this context. As a matter of fact, when preferences are separable the theory tells us the right behavior again, that is, voting sincerely on every single issue is the dominant strategy. When preferences are non-separable, instead, sincerity may lead to the worst outcome for everybody. Consider for example a referendum with three issues and three voters, and suppose that the worst alternative for everybody is that all the three issues pass together. Player 1 prefers that the first two issues pass to every other possible alternative, player 2 that the second and the third issues pass, while player 3 's best outcome is that the first and the third issues pass. Under sincerity every issue takes the majority, so every player's worst scenario occurs. Note that in this case the sincere strategy profile is not even a Nash equilibrium. Player 1 for instance cannot affect the decision on the third issue, which passes independently of her vote, while she is pivotal for the first two issues. Approving both of them is clearly not a best reply for her, as it would induce her worst alternative.

It is known that even focusing on Nash equilibria would be unsatisfactory in a voting context. In fact, with at least three players, for any outcome there is a Nash equilibrium inducing it, e.g. the strategy profile in which everybody approves that outcome so that nobody is pivotal. This is obviously true also in the single-issue case as well as in any two-candidate majority election. Even if in mass elections voters are typically not pivotal, in those cases the theory prescribes the use of dominant strategies due to the fact that there is always a slight probability of being decisive. This fact can be formally justified either assuming some sort of incomplete information or using solution concepts that embed some uncertainty in the actual strategy played by the opponents, as trembling-hand perfection à la Selten (1975).

In the multi-issue setting, a clear analysis of the problem under incomplete information is offered by Ahn and Oliveros (2012). In this paper we focus on the complete information case. Following the latter of the above two approaches, we provide a solution concept that introduces uncertainty in the form of possible mistakes that voters make when voting. Such a concept refines Nash equilibria in which players are not pivotal, eliminating those of them that do not satisfy an extremely simple and intuitive criterion.

We adopt a concept of sincerity, that we call "issue sincerity", which is based on the idea of conditional sincerity introduced by Alesina and Rosenthal (1996) in a con-

\footnotetext{
1 An example is given by the November 2016 California ballot, in which citizens have been asked to vote on seventeen issues, including marijuana legalization, gun control, drug prices, and condoms in porn.

2 In the example above, for instance, a voter may want marijuana legalization and gun control to pass together, but prefer neither issue to be implemented rather than each issue passing alone for fear of armed stoned people.
} 
text of multiple elections under complete information. In particular, given a reference outcome, the issue sincere strategy for a voter prescribes to vote sincerely issue by issue, considering as fixed the outcome of the others. An issue sincere strategy combination is a profile in which every voter's strategy is issue sincere given the outcome that such a combination induces. We show that the conditions for the existence of an issue sincere strategy combination are equivalent to the conditions for the existence of a local Condorcet winner, that is, an outcome that the majority prefers to every other alternative that differs in one issue only.

The concept of issue sincerity naturally arises from the idea that voters are typically not decisive and, even if there is a small probability that they are pivotal, being pivotal for more than one issue is much less probable than being pivotal for a single issue. We formalize this idea using a refinement of trembling-hand perfection that takes into account the material voting procedure. Roughly speaking, we limit the probability of making mistakes on a set of issues to be of smaller order than the probability of making mistakes on any of its subsets. We prove that such a solution concept coincides in pure strategies with the concept of issue sincere Nash equilibrium.

In voting theory it is standard to advocate the principle of stability against iterated elimination of dominated strategies, called "sophisticated voting" from Farquharson (1969) on. In this view, we analyze whether such a principle can eliminate an issue sincere Nash equilibrium. We define a simpler process, that we call "iterated determinacy of issues", which reduces a game fixing the outcome of an issue whenever it is determined by the undominated behavior of the majority of the voters. We show that every issue sincere equilibrium outcome survives this procedure, which coincides with iterated dominance except for very specific knife-edge cases.

To present the difficulties and the results in this voting context, we offer several examples characterized by few players and few issues. Nevertheless, any argument we give holds in general. Real-life multi-issue referenda typically involve a huge number of voters. To describe large electorates one could choose to specify every single voter's preferences, however this is not the most common approach. In the same vein as Debreu and Scarf (1963), we instead interpret a large electorate as the result of replicas of an original smaller one. We prove that for large elections an outcome is an issue sincere equilibrium outcome if and only if it is a local Condorcet winner. Moreover, we show that an issue sincere Nash equilibrium is stable against sophisticated voting, as iterated determinacy of issues and iterated dominance turn out to coincide.

Some political science papers have pointed out a few problems related to nonseparable preferences across multiple issues. Brams et al $(1997,1998)$ present several examples of non-separable preferences, and observe that if voters vote sincerely the winning outcome may receive the fewest votes, a phenomenon that they call "the paradox of multiple elections". Lacy and Niou (2000) show that under sincere voting a Condorcet loser can be the outcome of the election, and that both sincere and sophisticated voting can fail to select a Condorcet winner when it exists. This does not happen under sophisticated sequential voting. In our work we assume that voters vote strategically and simultaneously on the different issues and we propose a solution concept that also guarantees such positive results. 
Ahn and Oliveros (2012) consider strategic and simultaneous voting as well but under the assumption of incomplete information, and employ the solution concept of symmetric undominated Bayesian Nash equilibrium. They focus mostly on large elections with two issues and show that a Condorcet winner is not necessarily a limit outcome (that is, an outcome that is elected in equilibrium with probability converging to 1). In our setting, the equivalence between issue sincerity and the aforementioned version of perfection implies that uncertainty can be interpreted as introduced via strategy perturbations that are independent across issues. One consequence is that, differently from Ahn and Oliveros (2012), the probability that an issue passes does not change if it is conditioned on being pivotal for some other issue. This is the reason why we have the result that a Condorcet winner is always an issue sincere equilibrium outcome of large elections.

As mentioned above, we borrow the idea of sincerity from Alesina and Rosenthal (1996). In particular, they consider the simultaneous elections of the executive and the legislature and they denote with "conditional sincerity" the sincere vote in each election conditional on the result in the other one. ${ }^{3}$ Analogously, issue sincerity prescribes the sincere vote on each issue conditional on the resolution of the remaining ones. Conditional sincerity and independent trembles are employed also in De Sinopoli et al (2013) for the same type of simultaneous elections of the President and the Congress. Differently from our context, in their setting outcomes are represented over a unidimensional policy space and voters have single-peaked preferences so a Condorcet winner always exists, however it is not necessarily a conditionally sincere outcome.

The paper proceeds as follows. Section 2 describes the combinatorial game of voting on multiple issues. In Section 3 we characterize undominated strategies and analyze the concept of issue sincerity, showing the equivalence of local Condorcet winners and issue sincere outcomes. Section 4 presents the game-theoretical foundation of issue sincerity through a refinement of perfection, and Section 5 shows the stability of such a solution concept against iterated determinacy of issues. In Section 6 we characterize issue sincere equilibrium outcomes of large elections and we prove their robustness against iterated dominance.

\section{Preliminaries}

We begin by defining the class of combinatorial voting games. Let $N=\{1, \ldots, n\}$ be the finite set of players, where $n \geq 3$ is an odd number, and let $K$ be the finite set of binary issues. We denote with $\Omega$ the power set of $K$, that is, the set of possible outcomes. Under combinatorial voting the strategy set $S_{i}$ of player $i$ can be described as the collection of \#K-dimensional vectors with entries $s_{i}^{k} \in\{0,1\}$ for every $k \in$ $K$, where $s_{i}^{k}=1$ means that player $i$ approves issue $k$ while $s_{i}^{k}=0$ means that she does not. The set of strategy profiles is $S:=\prod_{i \in N} S_{i}$. Let $\theta: S \rightarrow \Omega$ be the outcome function. According to the combinatorial rule, the outcome induced by the strategy

\footnotetext{
3 Such a concept has been employed also in Ingberman and Rosenthal (1997).
} 
profile $s \in S$ is

$$
\theta(s):=\left\{k \in K: \#\left\{i \in N: s_{i}^{k}=1\right\} \geq \frac{n+1}{2}\right\},
$$

meaning that all the issues approved by more than half of the players pass. ${ }^{4}$

Each player $i$ has preferences $\gtrsim_{i}$ defined over the set of possible outcomes. For the sake of simplicity, we will always assume strict preference orders. A player's preferences can be separable in some (possibly every) issue. Separability in a given issue means that the player's preference for that issue to pass does not depend on the resolution of any other issue. With slight abuse of notation, we denote with $K^{\prime}$ the outcome where all and only the issues in $K^{\prime} \subseteq K$ pass ( $\varnothing$ denoting the outcome such that no issue passes).

Definition 1 Preferences $\gtrsim_{i}$ are positively separable in $k$ if $K^{\prime} \cup\{k\} \succ_{i} K^{\prime}$ for every $K^{\prime} \subseteq K \backslash\{k\}$, while they are negatively separable in $k$ if $K^{\prime} \succ_{i} K^{\prime} \cup\{k\}$ for every $K^{\prime} \subseteq K \backslash\{k\}$.

As usual, for player $i$ strategy $s_{i}$ is a best response to $s_{-i}$ if $\theta\left(s_{i}, s_{-i}\right) \gtrsim_{i} \theta\left(\tilde{s}_{i}, s_{-i}\right)$ for all $\tilde{s}_{i} \in S_{i}{ }^{5}$ The finite set of these strategies is written $\operatorname{PBR}_{i}\left(s_{-i}\right)$.

Definition 2 The strategy profile $s$ is a Nash equilibrium if $s_{i} \in \operatorname{PBR}_{i}\left(s_{-i}\right)$ for every $i$.

It is well known that the definition of Nash equilibrium allows the use of dominated strategies. Furthermore, as mentioned in the Introduction, when $n \geq 3$ every outcome can be supported by a Nash equilibrium. There is therefore need for equilibrium refinement also in combinatorial voting games. In the single-issue setting, undominance is guaranteed by sincerity. In fact, in that case the strategy combination in which every player votes sincerely is the unique undominated profile. Following the idea of conditional sincerity of Alesina and Rosenthal (1996), we define a concept of sincerity that seems appropriate in the context of multiple issues.

\section{Issue sincerity}

We want first to characterize the set of undominated strategies. The definition of (weak) dominance is standard.

Definition 3 Strategy $s_{i}$ weakly dominates strategy $\tilde{s_{i}}$ for player $i$ if

$$
\begin{array}{ll}
\theta\left(s_{i}, s_{-i}\right) \succsim_{i} \theta\left(\tilde{s}_{i}, s_{-i}\right) & \text { for all } s_{-i} \in S \backslash S_{i}, \\
\theta\left(s_{i}, s_{-i}\right) \succ_{i} \theta\left(\tilde{s}_{i}, s_{-i}\right) & \text { for at least one } s_{-i} \in S \backslash S_{i} .
\end{array}
$$

\footnotetext{
4 Oddness of the number of players is assumed just to guarantee that a pure strategy profile induces a pure outcome. In this case preference orders over outcomes are sufficient to study the main concepts that we introduce. We could replace this assumption with any deterministic tie-breaking rule.

5 A player's best response depends only on the aggregate behavior of the opponents, i.e., on the sum of the positive votes that each issue gets from the others. Henceforth, we will often use a vector to summarize this information, and we will refer to it as "pivotal event" if the player is decisive for some issue.
} 
Strategy $s_{i}$ is weakly dominant for player $i$ if it weakly dominates every other strategy $\tilde{s}_{i} \in S_{i}$. A strategy is weakly dominated for player $i$ if there exists another strategy that dominates it. Strictly dominated strategies do not typically appear in combinatorial voting games, and henceforth we will refer to weak dominance simply as dominance.

We can show that there exists the following relationship between dominance and separability. ${ }^{6}$

Proposition 1 For player i strategy $s_{i}$ is dominated by strategy $\tilde{s}_{i}$ if and only if, for all $k$ such that $s_{i}^{k} \neq \tilde{s}_{i}^{k}$,

(i) preferences $\gtrsim_{i}$ are separable in $k$ and

(ii) $\tilde{s}_{i}^{k}=1$ if and only if they are positively separable.

Proof If preferences of player $i$ are not separable in issue $k$, then there exist by definition two outcomes $K^{\prime}, K^{\prime \prime} \subseteq K \backslash\{k\}$ such that $K^{\prime} \cup\{k\} \succ_{i} K^{\prime}$ and $K^{\prime \prime} \succ_{i} K^{\prime \prime} \cup\{k\}$. Let $s_{i}^{k}=0$ and $\tilde{s}_{i}^{k}=1$. Consider the strategy profile of the opponents in which half of them approve all and only the issues in $K^{\prime}$ while the other half approve all and only the issues in $K^{\prime} \cup\{k\}$. Clearly, player $i$ strictly prefers strategy $\tilde{s}_{i}$ to strategy $s_{i}$. Analogously, we can construct a strategy profile of the opponents such that player $i$ strictly prefers $s_{i}$ to $\tilde{s}_{i}$. Therefore, neither $s_{i}$ can be dominated by $\tilde{s}_{i}$ nor viceversa, and hence whenever $s_{i}$ is dominated by $\tilde{s}_{i}$ preferences must be separable in all the issues in which the two strategies differ.

The if part is obvious, since either $s_{i}$ and $\tilde{s}_{i}$ are outcome equivalent or the outcome induced by $\tilde{s}_{i}$ is preferred by player $i$ to the outcome induced by $s_{i}$ due to (ii).

To get rid of equilibria in dominated strategies, one may simply focus on undominated Nash equilibria. However, the following example shows that such a solution concept may select every player's worst outcome.

Example 1 Consider a combinatorial voting game with three players and two issues, $A$ and $B$. By the combinatorial voting rule, all the issues that receive at least two positive votes pass. Players have the same preference order over the set of outcomes $\Omega=\{A, B, A B, \varnothing\}$, precisely $\varnothing \succ A B \succ A \succ B$. Note that these preferences are not separable either in $A$ or in $B$, so there is no dominated strategy by Proposition 1. It is easy to see that the strategy profile $s^{*}=((0,1),(0,1),(0,1))$, which prescribes every player to approve just issue $B$, is an undominated Nash equilibrium that induces the worst alternative for everybody.

Notice that $s^{*}$ is an equilibrium because nobody is pivotal. When a player has to decide how to vote, she believes that she is not going to be decisive on either issue, because $A$ takes no votes from the other players and $B$ already takes the majority. Of course, a vote on a given issue does never affect the outcome of any other issue. Thus, when deciding how to express her preferences for issue $A$, for example, a player can conceivably take as given the outcome of issue $B$. A fortiori in this case, where she is

\footnotetext{
${ }^{6}$ Lacy and Niou (2000) and Ahn and Oliveros (2012) observe that if preferences are separable in every issue then voting accordingly to the most preferred outcome is a dominant strategy for a player. This is an immediate corollary of the characterization in Proposition 1.
} 
not decisive on such an issue. Given that $B$ passes, a player should vote accordingly to her preferences over $A B$ and $B$, and hence should approve issue $A$ since she prefers $A B$ to $B$. Analogously, when deciding how to express her preferences for issue $B$, a player can consider as given the outcome of issue $A$, so she should never approve $B$ as she prefers $\varnothing$ to $B$. Therefore, a player who expects the others to approve just issue $B$ should reply approving just issue $A$. It follows that the equilibrium $s^{*}$ does not survive this intuitive reasoning, which we formalize with the concept of issue sincerity.

Definition 4 Strategy $s_{i}$ is issue sincere with respect to outcome $K^{\prime} \subseteq K$ if

(i) for every $k \in K^{\prime}, s_{i}^{k}=1$ if and only if $K^{\prime} \succ_{i} K^{\prime} \backslash\{k\}$, and

(ii) for every $k \notin K^{\prime}, s_{i}^{k}=1$ if and only if $K^{\prime} \cup\{k\} \succ_{i} K^{\prime}$.

The strategy profile $s$ is issue sincere if $s_{i}$ is issue sincere with respect to $\theta(s)$ for every $i$.

We say that an outcome is issue sincere if there is an issue sincere strategy profile that induces it. The strict preferences assumption implies that a player's issue sincere strategy with respect to a given outcome is unique. It is then immediate to find all the issue sincere outcomes of a combinatorial voting game. For instance, the game in Example 1 has two issue sincere outcomes, $\varnothing$ and $A B$. In fact, $s_{i}=(0,0)$ is every player's issue sincere strategy with respect to $\varnothing$ and $s_{i}=(1,1)$ the one with respect to $A B$. We have seen that $s_{i}=(1,0)$ is every player's issue sincere strategy with respect to outcome $B$, which therefore is not issue sincere as the corresponding issue sincere strategy profile induces outcome $A$. Similarly, $A$ is not an issue sincere outcome because every player's corresponding issue sincere strategy approves just issue $B$.

It readily follows from the characterization of dominated strategies in Proposition 1 that every issue sincere strategy is undominated. Unfortunately, an issue sincere strategy profile does not always exist, as the following example shows.

Example 2 Consider a three-player game with two issues, $A$ and $B$, where players' preferences are

$$
\begin{aligned}
& \varnothing \succ_{1} B \succ_{1} A B \succ_{1} A, \\
& A \succ_{2} \varnothing \succ_{2} B \succ_{2} A B, \\
& A B \succ_{3} A \succ_{3} B \succ_{3} \varnothing .
\end{aligned}
$$

It is easy to check that given any outcome the corresponding issue sincere strategy profile does not induce it. (Take for example outcome $A$. The corresponding issue sincere strategy profile is $s=((0,1),(1,0),(1,1))$ and hence induces outcome $A B$.)

Note that this game does not have a Condorcet winner, whose existence turns out to be a sufficient condition for the existence of an issue sincere outcome. However, in a combinatorial voting game it seems more appropriate to compare a given alternative with its "closest" ones, that is, those that differ in only one issue. Following Ahn and Oliveros (2012), we thus introduce the definition of local Condorcet winner.

Definition 5 Outcome $K^{\prime} \subseteq K$ is a local Condorcet winner if

$$
\begin{array}{ll}
\#\left\{i \in N: K^{\prime} \succ_{i} K^{\prime} \backslash\{k\}\right\}>\#\left\{i \in N: K^{\prime} \backslash\{k\} \succ_{i} K^{\prime}\right\} & \forall k \in K^{\prime}, \\
\#\left\{i \in N: K^{\prime} \succ_{i} K^{\prime} \cup\{k\}\right\}>\#\left\{i \in N: K^{\prime} \cup\{k\} \succ_{i} K^{\prime}\right\} & \forall k \notin K^{\prime} .
\end{array}
$$


We can now prove the following.

Proposition 2 An outcome is issue sincere if and only if it is a local Condorcet winner.

Proof Let $K^{\prime}$ be a local Condorcet winner and let $s$ be the corresponding issue sincere strategy profile. Consider an issue $k$. By definition, for every player $i$, if $k \in K^{\prime}$ then $s_{i}^{k}=1$ if and only if $K^{\prime} \succ_{i} K^{\prime} \backslash\{k\}$, while if $k \notin K^{\prime}$ then $s_{i}^{k}=1$ if and only if $K^{\prime} \cup\{k\} \succ_{i}$ $K^{\prime}$. Since $K^{\prime}$ is a local Condorcet winner, it follows that if $k \in K^{\prime}$ then $k \in \theta(s)$ while if $k \notin K^{\prime}$ then $k \notin \theta(s)$. Viceversa, let $K^{\prime}$ be an issue sincere outcome. For at least $\frac{n+1}{2}$ players, if $k \in K^{\prime}$ then $K^{\prime} \succ K^{\prime} \backslash\{k\}$ while if $k \notin K^{\prime}$ then $K^{\prime} \succ K^{\prime} \cup\{k\}$, so $K^{\prime}$ is a local Condorcet winner.

The above proposition directly implies that whenever each player has separable preferences a local Condorcet winner exists. As a matter of fact, a player's dominant strategy is issue sincere with respect to every outcome, so the outcome induced by the dominant strategy profile is a local Condorcet winner. ${ }^{7}$ The same is true when outcomes can be represented over a unidimensional space and preferences are singlepeaked.

The presence of a Condorcet winner is not sufficient to guarantee the existence of an issue sincere Nash equilibrium, as the following example shows.

Example 3 Consider a combinatorial voting game with two issues, where the three players' preferences are

$$
\begin{aligned}
& \varnothing \succ_{1} A B \succ_{1} A \succ_{1} B, \\
& B \succ_{2} A B \succ_{2} A \succ_{2} \varnothing, \\
& A \succ_{3} A B \succ_{3} B \succ_{3} \varnothing .
\end{aligned}
$$

The game has a Condorcet winner, $A B$, but the unique issue sincere strategy profile $s=((1,1),(0,1),(1,0))$, which induces it, is not a Nash equilibrium. Indeed, player 1 is pivotal for both issues and, therefore, her unique best reply against the opponents' aggregate behavior $(1,1)$ is $s_{1}=(0,0)$.

The reason why in this example the issue sincere strategy profile $s$ is not a Nash equilibrium is that player 1 is "double pivotal". In fact, when no player is pivotal for more than one issue then an issue sincere strategy profile is a Nash equilibrium. Obviously, if a player is not pivotal for any issue then any of her strategies is a best reply to the other players' aggregate behavior. On the other hand, if a player is pivotal just for one issue then her issue sincere strategy is a best reply, like every other

\footnotetext{
7 Separable preferences do not imply the existence of a Condorcet winner. Take for example three players with the following separable preferences:

$$
\begin{aligned}
& A \succ_{1} \varnothing \succ_{1} A B \succ_{1} B, \\
& B \succ_{2} \varnothing \succ_{2} A B \succ_{2} A, \\
& A B \succ_{3} A \succ_{3} B \succ_{3} \varnothing .
\end{aligned}
$$

The dominant strategy profile $s=((1,0),(0,1),(1,1))$ induces the local Condorcet winner $A B$, which however is not a Condorcet winner as the majority of the players prefer outcome $\varnothing$ to it.
} 
strategy that behaves in the same way in that issue. When a player is pivotal for more than one issue, instead, she can simultaneously alter the outcomes of those issues she is decisive for. Issue sincerity does not take into account such a possibility, since it prescribes to express preferences issue by issue, keeping fixed the outcome of the others. Therefore, in this case an issue sincere strategy may not be a best reply to the opponents' aggregate behavior, as in the previous example.

It follows that a sufficient condition for the existence of an issue sincere Nash equilibrium is the presence of a local Condorcet winner and the absence of players that are pivotal for more than one issue in the corresponding issue sincere strategy profile. The absence of such pivotality seems a very reasonable assumption in reallife referenda with a mass electorate, where even the probability of being pivotal for just one issue is practically negligible. In Section 6 we will see that if we interpret a large economy in the spirit of Debreu and Scarf (1963) as the result of replicas of a smaller original one, then pivotality for more than one issue disappears for large electorates. Thus, in large elections, when a Condorcet winner exists there is always an issue sincere Nash equilibrium that induces its election.

\section{A refinement of perfection}

In this section we show that the solution concept of issue sincerity has a gametheoretical foundation in a refinement of perfection, that we call " $b$-perfect equilibrium".

First, recall that the bad equilibrium $s^{*}$ of Example 1, which prescribes every player to approve just issue $B$, is undominated. It is well known that a refinement of the undominated Nash equilibrium concept is perfection.

Definition 6 The strategy profile $s$ is a perfect equilibrium if there exists a sequence of completely-mixed strategy profiles converging to $s$ such that $s$ is a best response to every element of such a sequence.

We can see that the strategy profile $s^{*}$ is not a perfect equilibrium. Take any sequence of completely-mixed strategy profiles that converges to it. Sufficiently close to $s^{*}$, every player faces the pivotal event $(2,1)$ induced by her opponents with a probability that is of smaller order than the probabilities of the pivotal events $(1,2)$ and $(1,1)$. In fact, the first event is the result of two deviations from $s^{*}$, because one opponent has to play strategy $(1,1)$ and the other has to play strategy $(1,0)$. On the contrary, the other two pivotal events are the result of just one of these deviations. Hence, close enough to $s^{*}$ every player strictly prefers strategy $(1,0)$ to strategy $(0,1)$, so $s^{*}$ is not perfect.

Even if in this case perfection eliminates an equilibrium that is not issue sincere, this does not hold in general, as the following example demonstrates.

Example 4 Consider a combinatorial voting game with three players and two issues, $A$ and $B$. Let preferences be

$$
\begin{aligned}
& \varnothing \succ_{i} B \succ_{i} A B \succ_{i} A, \quad i=1,2, \\
& A B \succ_{3} A \succ_{3} B \succ_{3} \varnothing .
\end{aligned}
$$


The first two players' preferences are negatively separable in $A$, while player 3's preferences are positively separable both in $A$ and in $B$. Hence, strategy $s_{3}=(1,1)$ is dominant for the third player.

Now, take the strategy profile $s^{\prime}=((0,1),(0,1),(1,1))$, which is a Nash equilibrium that induces outcome $B$. Note that the strategies of the first two players are not issue sincere. ${ }^{8}$ We can show that $s^{\prime}$ is a perfect equilibrium. To this end, consider the sequence of completely-mixed strategy profiles $\left\{s^{\varepsilon}\right\}_{\varepsilon \rightarrow 0}$ converging to $s^{\prime}$ such that

$$
\begin{aligned}
& s_{i}^{\varepsilon}=\left(1-\varepsilon-\varepsilon^{2}\right)(0,1)+\varepsilon(1,0)+\varepsilon^{2} \sigma^{\circ}, \quad i=1,2, \\
& s_{3}^{\varepsilon}=\left(1-\varepsilon^{2}\right)(1,1)+\varepsilon^{2} \sigma^{\circ},
\end{aligned}
$$

where $\sigma^{\circ}$ is an arbitrary completely-mixed strategy. For vanishing $\varepsilon$, players 1 and 2 face the pivotal event $(2,1)$ with a probability that is infinitely greater than the probability of any other possible event different from $(1,2)$. It follows that $s_{i}=(0,1)$ is a best response to $s_{-i}^{\varepsilon}$ for $i=1,2$. Thus, since $s_{3}=(1,1)$ is dominant for player 3 , $s^{\prime}$ is a perfect equilibrium.

To justify the perfection of $s^{\prime}$, the converging sequence of completely-mixed strategy profiles needs to be such that the deviations of the first two players from strategy $(0,1)$ to strategy $(1,0)$ are much more probable than any other possible deviation. ${ }^{9}$ If we interpret deviations as due to mistakes that players make when they vote, this seems unreasonable. As a matter of fact, the material procedure of voting requires each voter to cast two votes, one on issue $A$ and one on issue $B$, so the probability of making mistakes on both issues should be smaller than the probability of making a mistake on a single one. To capture this idea, we propose a refinement of perfection which puts further restrictions on the converging sequence of strategies.

Before giving the definition of such a solution concept, we need to introduce $b$ strategies. A $b$-strategy $b_{i}$ of player $i$ is a $\# K$-dimensional vector with entries $b_{i}^{k} \in$ $[0,1]$ for every $k \in K$, where $b_{i}^{k}$ represents the probability that player $i$ approves issue $k .{ }^{10} \mathrm{~A} b$-strategy profile is a vector $b=\left(b_{1}, \ldots, b_{n}\right)$. Let $b(s)$ be the $b$-strategy profile corresponding to the strategy profile $s$.

Definition 7 The pure strategy profile $s$ is a b-perfect equilibrium if there exists a sequence of completely-mixed $b$-strategy profiles converging to $b(s)$ such that $s$ is a best response to every element of such a sequence.

\footnotetext{
8 The issue sincere strategy profile with respect to outcome $B$ is $s=((0,0),(0,0),(1,1))$, so $B$ is not an issue sincere outcome.

9 If every player plays according to $s^{\prime}$, strategies $(0,0)$ and $(0,1)$ are equivalent for the first two players and they are strictly better than strategies $(1,0)$ and $(1,1)$, which would induce outcome $A B$. Hence, closeby $s^{\prime}$, the first two strategies are the only best replies for them. Clearly, these strategies induce a different outcome only when the first two players are pivotal for issue $B$, that is, $B$ takes one vote from the opponents. In this case, if issue $A$ takes two votes then they strictly prefer strategy $(0,1)$, otherwise they strictly prefer strategy $(0,0)$. For $s^{\prime}$ to be perfect, then, the probability of the pivotal event $(2,1)$ has to be sufficiently greater than the probability of the events $(0,1)$ and $(1,1)$.

10 We use the term $b$-strategy as a reminiscence of behavioral strategy. In fact, $b$-strategies would precisely be behavioral strategies if we described a combinatorial voting game as an extensive form one.
} 
Every completely-mixed $b$-strategy profile has a corresponding completely-mixed strategy profile. ${ }^{11}$ Thus, every $b$-perfect equilibrium is perfect. The converse is not true. In fact, we now prove that the issue sincere equilibrium concept coincides with $b$-perfection in pure strategies.

Proposition 3 A pure strategy profile is a b-perfect equilibrium if and only if it is an issue sincere Nash equilibrium.

Proof Let $s$ be a $b$-perfect equilibrium. Clearly, every best response of player $i$ to $s_{-i}$ behaves issue sincerely in all the issues $k$ for which player $i$ is pivotal. So consider an issue $k^{\prime}$ player $i$ is not decisive for. In any completely-mixed $b$-strategy profile close to $b(s)$, the probability that $i$ is pivotal for $k^{\prime}$ and the outcome of all the other issues is the one induced by $s$ is of greater order than the probability that $i$ is pivotal for $k^{\prime}$ and the resolution of the other issues is different. Thus, every best reply of player $i$ must necessarily behave issue sincerely also in $k^{\prime}$ for $s$ to be $b$-perfect. It follows that $s$ is an issue sincere Nash equilibrium.

To prove the converse, note that the above reasoning implies that, given an issue sincere Nash equilibrium, a player's issue sincere best reply is the only best reply to a close-by completely-mixed $b$-strategy profile.

From Proposition 3 it follows that a sufficient condition for the existence of a $b$ perfect equilibrium in pure strategies is the presence of a local Condorcet winner and the absence of players that are pivotal for more than one issue in the corresponding issue sincere strategy profile.

In the Appendix we show that a $b$-perfect equilibrium may not exist even if we extend its definition to mixed $b$-strategies. In fact, this is equivalent to the original definition of perfect equilibrium by Selten (1975) for extensive-form games, but without perfect recall. Think about the actual process of filling the ballot in a multi-issue referendum, which requires a voter to mark the ballot paper several times. If she makes a mistake when voting on a given issue and notices it, she can ask for a new ballot paper and mark it again correctly. Hence, to model properly the possibility of making mistakes, when a voter is voting for an issue she must not know if she has made a mistake on a previous one; that is, there is not perfect recall, and trembles are independent across issues. While a perfect equilibrium always exists in an extensive-form game with perfect recall, this is not the case otherwise. ${ }^{12}$ In particular, the reason is that the best reply correspondence is not convex-valued in the $b$-strategy space.

\section{Iterated dominance}

Iterated dominance has a longstanding tradition in voting theory, where it has been introduced by Farquharson (1969) through the concept of sophisticated voting. Like-

\footnotetext{
11 In particular, to the $b$-strategy $(x, y)$ with $x, y \in(0,1)$ corresponds the completely-mixed strategy $x y(1,1)+x(1-y)(1,0)+y(1-x)(0,1)+(1-x)(1-y)(0,0)$.

12 This result is in general not new, as Wichardt (2008) shows that an extensive-form game without perfect recall may not have any Nash equilibrium in behavioral strategies. However, his example cannot be framed in the context of combinatorial voting.
} 
wise, it has been advocated by prominent game theorists as a basic principle that a solution concept should satisfy (Kohlberg and Mertens, 1986).

Before analyzing the relationship between iterated dominance and issue sincerity, we present an example to better highlight some features of iterated dominance in our setting.

Example 5 Consider the combinatorial voting game with issues $A$ and $B$, where three players have preferences given by

$$
\begin{aligned}
& A B \succ_{i} B \succ_{i} \varnothing \succ_{i} A, \quad i=1,2, \\
& \varnothing \succ_{3} A B \succ_{3} A \succ_{3} B .
\end{aligned}
$$

By Proposition 1, player 3 does not have any dominated strategy, while players 1 and 2 have two dominated strategies, $(1,0)$ and $(0,0)$, as their preferences are positively separable in $B$. In the reduced game obtained by eliminating these two strategies for both players, issue $B$ passes for sure. Therefore, up to duplications of strategies, this reduced game is equivalent to a single-issue game on $A$, where every player approves $A$ in every undominated strategy profile since every player prefers outcome $A B$ to outcome $B$. It follows that iterated dominance isolates outcome $A B$, which is the only issue sincere equilibrium outcome of the game. ${ }^{13}$

The game in this example is solvable by iterated dominance because some issue, viz. $B$, is determinate in the reduced game. This is due to the fact that enough players (two out of three) have all positively separable preferences in that issue. When there are too few players who have preferences separable in the same issue and in the same direction, a construction analogous to the one in the proof of Proposition 1 shows that there cannot be dominated strategies in the reduced game. Given this consideration we define a weaker version of iterated dominance, that we call "iterated determinacy of issues".

For every issue $k \in K$, let $n_{k}^{1}$ be the number of players with preferences positively separable in $k$, and $n_{k}^{0}$ be the number of players with preferences negatively separable in $k$. Moreover, let $K^{1} \subseteq K$ be the set of issues $k$ such that $n_{k}^{1} \geq \frac{n+1}{2}$, and $K^{0} \subseteq K$ be the set of issues $k$ such that $n_{k}^{0} \geq \frac{n+1}{2}$. We say that issue $k$ is determinate if it belongs to $K^{1} \cup K^{0}$. In fact, the characterization of dominated strategies in Proposition $1 \mathrm{im}$ plies that in this case the outcome of $k$ is univocally determined by the undominated behavior of the players.

Given an $n$-player combinatorial voting game $\Gamma$ with set of issues $K$, whenever $K^{1} \cup K^{0} \neq \emptyset$ we can define the reduced game $\Gamma^{\prime}$ as the $n$-player combinatorial voting game with set of issues $K^{\prime} \equiv K \backslash\left\{K^{1} \cup K^{0}\right\}$ and preferences induced by those in $\Gamma$ over the remaining outcomes. The process of iterated determinacy of issues consists in iteratively reducing a game as long as some issue is determinate. Then, a game is solvable by iterated determinacy of issues if at the end of the process every issue $k \in K$ is determinate.

13 The only strategies that survive iterated dominance are strategy $(1,1)$ for players 1 and 2 and strategies $(1,1)$ and $(1,0)$ for player 3 . 
The next proposition readily follows from the fact that in every reduced game the characterization of dominated strategies in Proposition 1 still holds, and hence every issue sincere strategy is undominated.

Proposition 4 An issue sincere Nash equilibrium is robust against iterated determinacy of issues. Thus, if a combinatorial voting game is solvable by iterated determinacy of issues then the outcome that survives is the unique issue sincere equilibrium outcome of the game.

Robustness against iterated determinacy of issues is a weaker requirement than robustness against iterated dominance. In fact, the two processes can behave differently only if, at some step, the number of players who have preferences separable in the same issue and in the same direction is exactly equal to $\frac{n-1}{2}$. In this very specific case such an issue is not determinate, and hence the first process cannot univocally fix it and continue. However, iterated dominance may go on, because in the game obtained after eliminating dominated strategies some dominated strategy may exist even if preferences are not separable. The reason is that, in such a reduced game, opponents' strategy combinations that determine a given outcome of a given issue do not necessarily exist. Therefore, iterated dominance can eliminate an outcome that survives iterated determinacy of issues, as it is shown in the following example.

Example 6 Consider the three-player combinatorial voting game with issues $A$ and $B$, where preferences are

$$
\begin{aligned}
& A B \succ_{i} \varnothing \succ_{i} A \succ_{i} B, \quad i=1,2, \\
& \varnothing \succ_{3} A \succ_{3} B \succ_{3} A B .
\end{aligned}
$$

Since the first two players do not have separable preferences, neither issue $A$ nor issue $B$ is determinate and all the outcomes survive iterated determinacy of issues.

Player 3 has a dominant strategy, $(0,0)$. Therefore, after the first round of elimination of dominated strategies, players 1 and 2 can face only four possible events, precisely $(0,0),(0,1),(1,0)$, and $(1,1)$. Strategies $(0,1)$ and $(1,0)$ become then dominated by strategy $(0,0)$ for them. Indeed, the pivotal events $(1,2)$ and $(2,1)$, in which they prefer approving respectively only issue $A$ and only issue $B$ to not approving any issue, are not realizable in such a reduced game. After eliminating also those strategies, players 1 and 2 can face either the event $(0,0)$ or the event $(1,1)$, and hence strategy $(0,0)$ becomes dominated by strategy $(1,1)$ for them. It follows that iterated dominance isolates a unique outcome, $A B$.

This example shows that an issue sincere equilibrium outcome may not survive iterated dominance. Indeed, it is easy to see that outcome $\varnothing$ is issue sincere, and it is induced by the Nash equilibrium $s=\{(0,0),(0,0),(0,0)\} .{ }^{14}$ The reason why it does not survive is that, after the second round of elimination of dominated strategies, the first two players face only the event in which they are double pivotal and the one in

\footnotetext{
14 In particular, outcome $\varnothing$ is a local Condorcet winner of the game, while the outcome that survives the process, $A B$, is the Condorcet winner. See the example in Table 5 in Lacy and Niou (2000) for a game in which iterated dominance eliminates the Condorcet winner and selects a local Condorcet winner.
} 
which they are not pivotal at all. Issue sincerity does not take into account the possibility of simultaneously altering the outcomes of two or more issues, and therefore an issue sincere strategy may become dominated after the deletion of some dominated strategies.

In the next section we will see that in large elections iterated dominance and iterated determinacy of issues are completely equivalent, so an issue sincere Nash equilibrium is always robust against sophisticated voting.

\section{Large elections}

Edgeworth (1881) introduced a standard procedure for enlarging an economy, which has become popular after the renowned contribution of Debreu and Scarf (1963). In particular, an economy is imagined to be composed of $n$ types of agents, with $m$ agents of each type. For two agents to be of the same type, it is required that they have the same characteristics (precisely, the same preferences and initial endowments). The economy consists therefore of $m n$ agents. A large economy is then interpreted as the result of replicas of an original one where there are exactly $n$ agents, which is made large by adding agents of the same type and in the same proportion as those already in it.

This is a standard approach to enlarge the number of agents also in models with incomplete information, as the one of Ahn and Oliveros (2012). In fact, in their setting voters are identical ex-ante, that is, their types are realized independently from the same distribution. Enlarging the number of players can therefore be seen as making replicas of the same "prototype" player.

In the same spirit, we say that an electorate is large if it can be obtained through replicas of an original smaller one. For each voter of a given electorate, in the $m$ replica electorate there will be $m$ voters with the same preferences, where $m$ is odd and at least equal to 3 to preserve the oddness assumption. With large electorates, the difficulties that emerge in the analysis of multi-issue referenda disappear and further positive results hold.

In Section 3, we saw that the presence of a local Condorcet winner may not be sufficient to guarantee the existence of an issue sincere Nash equilibrium. When some player is pivotal for more than one issue, indeed, her issue sincere strategy may not be optimal, as strategy $(1,1)$ of player 1 in Example 3. Now, take again that example and consider a replica of the voters, that is, assume that there are three voters who have the same preferences as player $i$, for $i=1,2,3$. It is clear that $A B$ is still the Condorcet winner, but now the issue sincere strategy profile inducing it is such that no player is pivotal, and hence it is a Nash equilibrium.

The disappearance of pivotality with replicas is a general phenomenon. In fact, the first replica of any group of voters is enough for no player to be pivotal in any strategy profile in which all the players with the same preferences choose the same strategy. It follows that the issue sincere strategy combination inducing the issue sincere outcome of Proposition 2 is a Nash equilibrium. We can therefore state the following. 
Proposition 5 In large elections, an outcome is an issue sincere equilibrium outcome if and only if it is a local Condorcet winner.

From Proposition 3 it directly follows that in large elections a local Condorcet winner is a $b$-perfect equilibrium outcome. In the context of incomplete information and two issues as modeled in Ahn and Oliveros (2012), a Condorcet winner may not be a limit outcome, that is, an outcome that is elected in equilibrium with probability converging to 1 as the number of players tends to infinity. In that setting, a distinction must be made between unconditional and conditional certainty of an issue. Both definitions indicate that, given a (symmetric) strategy profile, the probability that an issue passes converges either to 0 or to 1 , the latter probability being conditioned on the fact that a voter is pivotal for the other issue. Unconditional and conditional probabilities can diverge for some strategy combinations. Ahn and Oliveros (2012) show that a sufficient condition for a local Condorcet winner to be a limit outcome is that at least one issue conditionally agrees with it.

When uncertainty is introduced through perturbations of $b$-strategies, limits of probabilities can be defined for vanishing perturbations in the converging sequence of completely-mixed $b$-strategy combinations. Indeed, if we limit ourselves to pure strategy profiles, the conditional probability for a voter that an issue passes is welldefined only if the voter is pivotal for the conditioning issue. In our context, then, "unconditional certainty" implies "conditional certainty". ${ }^{15}$ Consider two issues $k$ and $k^{\prime}$, and let $\theta(s)$ be the outcome induced by the strategy combination $s$. Given a sequence of completely-mixed $b$-strategy profiles converging to $s$, the probability of the event in which a voter is pivotal for $k$ and the outcome of $k^{\prime}$ disagrees with $\theta(s)$ is of smaller order than the probability of the event in which that voter is pivotal for $k$ and the outcome of $k^{\prime}$ agrees with $\theta(s)$. Hence, the conditional probability that an issue passes converges to 1 if it is unconditionally certain to pass, and to 0 if it is unconditionally certain to fail. This means that, given a local Condorcet winner and the issue sincere strategy combination that induces it, for every voter all the issues conditionally agree with that winner, which is therefore a $b$-perfect equilibrium outcome.

In Section 5, we saw that an issue sincere equilibrium outcome may not survive iterated dominance, because an issue sincere strategy can become dominated in the game obtained after eliminating some dominated strategy. Recall that the only case in which this can happen is when exactly $\frac{n-1}{2}$ voters have preferences separable in the same issue and in the same direction. This is the case of Example 6, where one player out of three has preferences negatively separable both in issue $A$ and in issue $B$. Now, consider a replica of the game in that example, in which there are nine players. The number of voters who have preferences negatively separable in both issues becomes three, which is now smaller than the new threshold 4 , and therefore iterated dominance does no more eliminate any outcome.

We can easily show that iterated determinacy of issues and iterated dominance are equivalent for any replica of the electorate. To this end, take a group of $n$ voters for

\footnotetext{
15 The same relation would hold if uncertainty were introduced in the vote counting. As a matter of fact, under our assumption of strict preferences an equivalent definition of $b$-perfection could be stated based on a vanishing probability of misrecording votes, in a way similar to Laslier and Van der Straeten (2016).
} 
which issue $k$ is determinate. Clearly, in any m-replica electorate such an issue is determinate as well, since $n_{k}^{j} \geq \frac{n+1}{2}$ implies $m n_{k}^{j}>\frac{m n+1}{2}$ for $j=0,1$. On the other hand, if an issue is not determinate for the original group of voters then it is not determinate for any $m$-replica either, since $n_{k}^{j} \leq \frac{n-1}{2}$ implies $m n_{k}^{j}<\frac{m n-1}{2}$ for $j=0,1$. Thus, even if in the original electorate there are exactly $\frac{n-1}{2}$ voters with preferences separable in the same issue and in the same direction, in the m-replica electorate the number of those voters does not reach the new threshold. It follows that iterated determinacy of issues and iterated dominance coincide in large elections. Given Proposition 4, we can conclude the following.

Proposition 6 In large elections, an issue sincere Nash equilibrium is robust against iterated dominance. Thus, if a combinatorial voting game is dominance solvable then the outcome that survives is the unique issue sincere equilibrium outcome of the game.

\section{A Non-existence of b-perfect equilibria}

In this Appendix it is shown that, even if we allow players to use mixed $b$-strategies, a $b$-perfect equilibrium may not exist.

Consider the following extension of the $b$-perfect equilibrium concept to mixed $b$-strategies.

Definition 8 The $b$-strategy profile $b$ is a $b$-perfect equilibrium if there exists a sequence of completelymixed $b$-strategy profiles converging to $b$ such that $b$ is a best response to every element of such a sequence.

Similarly to perfection, an analogous definition of $b$-perfection can be stated in terms of perturbed games (Selten, 1975), generated by perturbing an original game in which every player's strategy set coincides with the set of $b$-strategies. Given that such a set is compact, a standard proof for the existence of a $b$-perfect equilibrium would require every perturbed game to have a Nash equilibrium in $b$-strategies. However, this is not always the case, because the best reply correspondence is not convex-valued in the $b$-strategy space. To see this, take a player with preferences $A B \succ \varnothing \succ A \succ B$ and utility values $u(A B)=10$, $u(\varnothing)=9, u(A)=3, u(B)=0$. Consider her best response against the strategy combination of two opponents in which one plays the pure strategy $(0,0)$ and the other plays the mixed strategy $\frac{9}{10}(1,1)+\frac{1}{10}(0,1)$. Strategies $(1,1)$ and $(0,0)$ are both best replies for that player, and they are strictly preferred to strategies $(1,0)$ and $(0,1)$. Nevertheless, every strictly convex combination between the two pure best replies in the space of $b$-strategies, i.e. every $b$-strategy $(x, x)$ with $0<x<1$, is strictly worse for the player and, hence, it is not a best reply. ${ }^{16}$

We now present an example in which a $b$-perfect equilibrium does not exist, neither in pure nor in mixed $b$-strategies. Recall that a $b$-perfect equilibrium is also perfect and hence undominated, since to every completely-mixed $b$-strategy corresponds a completely-mixed strategy. Therefore, for the sake of completeness, we describe also the sets of undominated and perfect equilibria of the game.

Example 7 Consider the combinatorial voting game with two issues $A$ and $B$ and three players, whose utility values are

$$
\begin{array}{llll}
u_{1}(A)=10, & u_{1}(B)=6, & u_{1}(\varnothing)=1, & u_{1}(A B)=0, \\
u_{2}(A)=3, & u_{2}(B)=0, & u_{2}(\varnothing)=9, & u_{2}(A B)=10, \\
u_{3}(A)=5, & u_{3}(B)=2, & u_{3}(\varnothing)=10, & u_{3}(A B)=0 .
\end{array}
$$

16 Note that to the $b$-strategy $(x, x)$ corresponds the mixed strategy $x^{2}(1,1)+x(1-x)(1,0)+x(1-$ $x)(0,1)+(1-x)^{2}(0,0)$, which gives positive weight also to the pure strategies that are not best replies. 
As usual, let $\sum_{i}$ be player $i$ 's set of mixed strategies and let $\sum:=\prod_{i \in N} \sum_{i}$. To simplify notation, let also

$$
\begin{array}{llll}
\sigma_{1}((1,0))=x, & \sigma_{1}((0,1))=y, & \sigma_{1}((0,0))=z, & \sigma_{1}((1,1))=1-x-y-z \\
\sigma_{2}((1,0))=p, & \sigma_{2}((0,1))=q, & \sigma_{2}((0,0))=r, & \sigma_{2}((1,1))=1-p-q-r
\end{array}
$$

It can be easily seen that strategy $s_{3}=(0,0)$ is dominant for player 3 and, therefore, it will be played with probability 1 in every undominated (and perfect) equilibrium. Let

$$
\begin{aligned}
& \hat{\Sigma}=\left\{\sigma \in \Sigma: 1-7 x-10 y-z \leq 0, r=1, \sigma_{3}((0,0))=1\right\}, \\
& \hat{\Sigma}^{p}=\{\sigma \in \hat{\Sigma}: z=0\} .
\end{aligned}
$$

Note that all the elements of both these sets induce outcome $\varnothing$. We can now show the following.

Proposition $7 \hat{\Sigma}$ and $\hat{\Sigma}^{p}$ are respectively the set of undominated Nash equilibria and the set of perfect equilibria of the game. The game does not have any b-perfect equilibrium.

Proof First, note that all the elements of $\hat{\Sigma}$ are equilibrium points, since player 1 is indifferent among all the four pure strategies and the condition $1-7 x-10 y-z \leq 0$ guarantees that strategy $(0,0)$ is a best reply for player $2 .{ }^{17}$ To see that no other undominated Nash equilibrium exists, we have to consider four possible cases.

a) Both player 1 and player 2 play a pure strategy. If $s_{2}=(0,0)$, the only undominated equilibria in pure strategies are the points in $\hat{\Sigma}$ corresponding to $x=1, y=1$, and $z=1$. If $s_{2}=(0,1)$ then player 1 has two pure best replies, $(1,1)$ and $(0,1)$. However, player 2 has a unique best reply against $(1,1)$, strategy $(1,1)$, while her pure best replies against $(0,1)$ are strategies $(0,0)$ and $(1,0)$. Similarly, if $s_{2}=(1,0)$ then player 1 has two pure best replies, $(1,1)$ and $(1,0)$, but player 2's pure best replies against $(1,0)$ are strategies $(0,0)$ and $(0,1)$. If $s_{2}=(1,1)$, player 1 's unique best reply is $(1,0)$, so again player 2 would rather prefer either strategy $(0,0)$ or strategy $(0,1)$. It follows that all the undominated Nash equilibria in pure strategies belong to the set $\hat{\Sigma}$.

b) Player 1 plays a pure strategy and player 2 plays a mixed strategy. If $s_{1}=(1,0)$, the pure best replies of player 2 are $(0,0)$ and $(0,1)$ but then, since she is completely mixing between these strategies, player 1 will strictly prefer strategy $(0,1)$ to strategy $(1,0)$. Likewise, if $s_{1}=(0,1)$ then the pure best replies of player 2 are $(0,0)$ and $(1,0)$, so player 1 will strictly prefer strategy $(1,0)$ to strategy $(0,1)$. If $s_{1}=(0,0)$, player 2 is indifferent among all the four pure strategies and, since $r \neq 1$, player 1 will then prefer to play either strategy $(1,0)$ or strategy $(0,1)$. Finally, if $s_{1}=(1,1)$ then player 2 has a unique best reply, $(1,1)$. Thus, no undominated Nash equilibrium of this kind exists.

c) Player 1 plays a mixed strategy and player 2 plays a pure strategy different from $(0,0)$. If $s_{2}=(1,0)$, player 1 should completely mix between her pure best replies $(1,0)$ and $(1,1)$, but in this case player 2 would strictly prefer strategy $(1,1)$ to strategy $(1,0)$. Analogously, if $s_{2}=(0,1)$ then player 1 's best replies are $(0,1)$ and $(1,1)$, so player 2 will strictly prefer strategy $(1,1)$ to strategy $(0,1)$. Lastly, if player 2 plays $s_{2}=(1,1)$ then player 1 has a unique best reply, strategy $(1,0)$. It follows that there does not exist any undominated Nash equilibrium of this kind either.

d) Both player 1 and player 2 play a mixed strategy. We can consider three cases:

i) Player 1 mixes between two pure strategies. When $x+y=1$ or $x=y=0$ player 2 has a unique best reply, $(0,0)$ and $(1,1)$ respectively. If $x+z=1$, player 2's pure best replies are $(0,0)$ and $(0,1)$, but then player 1 strictly prefers strategy $(0,1)$ to both strategies $(1,0)$ and $(0,0)$ against any mixed strategy of the opponent. Similarly, if $y+z=1$ then player 2 should completely mix between strategies $(0,0)$ and $(1,0)$, but player 1 then would strictly prefer to play strategy $(1,0)$. When $y=z=0$ or $x=z=0$ strategies $(1,0)$ and $(0,1)$ are never best replies for player 2, who thus will mix only between strategies $(0,0)$ and $(1,1)$. But in this case player 1 has a strict best reply, strategy $(1,0)$.

\footnotetext{
17 If player 3 plays $(0,0)$, player 2 weakly prefers strategy $(0,0)$ to both strategies $(1,0)$ and $(0,1)$. The condition on player 1 's strategy assures that player 2 prefers strategy $(0,0)$ also to strategy $(1,1)$ (i.e., $9 \geq 3 x+9 z+10(1-x-y-z))$.
} 
ii) Player 1 mixes between three pure strategies. When player 1 does not play strategy $(1,1)$ player 2 has a unique best reply, strategy $(0,0)$. In all the other cases, strategies $(1,0)$ and $(0,1)$ are never best replies for player 2. Again, if she mixes between strategies $(0,0)$ and $(1,1)$ then player 1 has a strict best reply, strategy $(1,0)$.

iii) Player 1 plays a completely-mixed strategy. Also in this case strategies $(1,0)$ and $(0,1)$ are never best replies for player 2 and then strategy $(1,0)$ is the unique best reply for player 1 .

Thus, $\hat{\Sigma}$ is the set of all the undominated Nash equilibria of the game and, hence, it contains the set of perfect equilibria. We can show that such a set is $\hat{\Sigma}^{p}$.

First, note that for any sequence of mixed strategies of players 2 and 3 converging to $(0,0)$, player 1 strictly prefers both strategies $(1,0)$ and $(0,1)$ to strategy $(0,0) \cdot{ }^{18}$ In fact, strategy $(0,0)$ is strictly preferred to strategy $(1,0)$ (resp. $(0,1))$ only against the pivotal event $(1,2)$ (resp. $(2,1))$, whose probability converges to 0 much faster than the probability of the pivotal event $(1,1)$, in which player 1 strictly prefers strategy $(1,0)$ (resp. $(0,1))$ to strategy $(0,0)$. Therefore, in every perfect equilibrium $z=0$.

To see that every element of $\hat{\Sigma}^{p}$ is perfect, fix $\hat{\sigma}^{p} \in \hat{\Sigma}^{p}$ and consider the sequence $\left\{\sigma^{\varepsilon}\right\}_{\varepsilon \rightarrow 0}$ of completely-mixed strategy profiles such that

$$
\begin{aligned}
& \sigma_{1}^{\varepsilon}=\left(1-\varepsilon^{1 / 3}\right) \hat{\sigma}_{1}^{p}+\varepsilon^{1 / 3}\left(\left(1-3 \varepsilon^{1 / 3}\right)(0,1)+\varepsilon^{1 / 3}(0,0)+\varepsilon^{1 / 3}(1,0)+\varepsilon^{1 / 3}(1,1)\right) \\
& \sigma_{2}^{\varepsilon}=(1-\alpha(\varepsilon)-\beta(\varepsilon)-\varepsilon)(0,0)+\alpha(\varepsilon)(1,0)+\beta(\varepsilon)(0,1)+\varepsilon(1,1) \\
& \sigma_{3}^{\varepsilon}=(1-3 \varepsilon)(0,0)+\varepsilon(1,0)+\varepsilon(0,1)+\varepsilon(1,1)
\end{aligned}
$$

where

$$
\begin{aligned}
& \alpha(\varepsilon)=\frac{\varepsilon+8 \varepsilon^{2}-13 \varepsilon^{3}}{3\left(1-8 \varepsilon+11 \varepsilon^{2}\right)}, \\
& \beta(\varepsilon)=\frac{3 \varepsilon-16 \varepsilon^{2}+17 \varepsilon^{3}}{1-8 \varepsilon+11 \varepsilon^{2}} .
\end{aligned}
$$

The values of $\alpha(\varepsilon)$ and $\beta(\varepsilon)$ assure that, for $\varepsilon$ close to 0 , player 1 is indifferent among strategies $(0,0)$, $(1,0)$, and $(0,1)$, and strictly prefers them to strategy $(1,1) \cdot{ }^{19}$ The perturbations of player 1 and player 3 's strategies, instead, guarantee that $(0,0)$ is the unique best reply for player 2 . Indeed, the probabilities of the pivotal events $(2,1)$ and $(1,2)$ are of smaller order than the probabilities of the pivotal events $(0,1)$ and $(1,0)$, so player 2 strictly prefers strategy $(0,0)$ to both strategies $(0,1)$ and $(1,0)$. Moreover, the probability of the pivotal event $(1,1)$ is not enough to make strategy $(1,1)$ preferred to strategy $(0,0)$ for her. Since the above sequence converges to $\hat{\sigma}^{p}$ as $\varepsilon$ goes to 0 , we have that $\hat{\sigma}^{p}$ is a perfect equilibrium.

From Definition 8, however, it readily follows that no point in $\hat{\Sigma}^{p}$ can be $b$-perfect. As a matter of fact, for any sequence of completely-mixed $b$-strategies of players 2 and 3 converging to $(0,0)$, player 1 faces the pivotal events $(0,1)$ and $(1,0)$ with a probability that is infinitely larger than the probability of any other event different from $(0,0)$. Her only best reply to every element of such a sequence is then strategy $(1,1)$. Therefore, we can conclude that there does not exist any $b$-perfect equilibrium in the game.

\section{References}

Ahn DS, Oliveros S (2012) Combinatorial voting. Econometrica 80(1):89-141

Alesina A, Rosenthal H (1996) A theory of divided government. Econometrica 64(6):1311-41

Brams SJ, Kilgour DM, Zwicker WS (1997) Voting on referenda: the separability problem and possible solutions. Electoral Studies 16(3):359 - 377

Brams SJ, Kilgour DM, Zwicker WS (1998) The paradox of multiple elections. Social Choice and Welfare 15(2):211-236

18 Among the equivalent definitions of perfect equilibrium proposed in the literature, we will use the extension of Definition 6 to mixed strategies (obtained just substituting in that definition $s$ with $\sigma$ ).

19 In particular, the utility of the first three strategies is $\frac{112 \varepsilon-992 \varepsilon^{2}+2352 \varepsilon^{3}-1664 \varepsilon^{4}}{3\left(1-8 \varepsilon+11 \varepsilon^{2}\right)}$, while the utility of strategy $(1,1)$ is $\frac{22 \varepsilon-92 \varepsilon^{2}-78 \varepsilon^{3}+316 \varepsilon^{4}}{3\left(1-8 \varepsilon+11 \varepsilon^{2}\right)}$. 
De Sinopoli F, Ferraris L, Iannantuoni G (2013) Electing a parliament. Social Choice and Welfare 40(3):715-737

Debreu G, Scarf H (1963) A limit theorem on the core of an economy. International Economic Review 4(3):235-246

Edgeworth FY (1881) Mathematical Psychics. London: Kegan Paul

Farquharson R (1969) Theory of Voting. New Haven: Yale University Press

Ingberman DE, Rosenthal H (1997) Median voter theorems for divisible governments. New York: Mimeo

Kohlberg E, Mertens JF (1986) On the strategic stability of equilibria. Econometrica 54(5):1003-37

Lacy D, Niou EM (2000) A problem with referendums. Journal of Theoretical Politics 12(1):5-31

Laslier JF, Van der Straeten K (2016) Strategic voting in multi-winner elections with approval balloting: a theory for large electorates. Social Choice and Welfare 47(3):559-587

Selten R (1975) Reexamination of the perfectness concept for equilibrium points in extensive games. International Journal of Game Theory 4(1):24-55

Wichardt PC (2008) Existence of nash equilibria in finite extensive form games with imperfect recall: A counterexample. Games and Economic Behavior 63(1):366 - 369 